\title{
Aflatoxin and Its Toxic Tragedies in Kenya
}

\author{
Kaiming $\operatorname{Tan}^{1 *}$
}

Aflatoxin is a mycotoxin produced by fungi, primarily Aspergillus flavus. Aspergillus flavus is often found in maize and peanuts. Aflatoxin is a serious health concern, especially in countries such as Kenya, where improper crop storage in warm, humid conditions causes it to thrive. In this review, we analyze the possible reasons behind the 2004 aflatoxin outbreak in Kenya. We also discuss the lessons learned from the tragedy and put forth potential solutions to prevent future outbreaks. Aflatoxin control is important to minimize crop damage and its adverse health effect on humans.

\section{INTRODUCTION}

For decades, mycotoxins have had serious public health threats and economic impacts in agriculture, especially in Kenya, China and India (Wu and Khlangwiset, 2010). Among these mycotoxins, aflatoxin is one of the most well-known and medically significant. Aflatoxin is a hepatocarcinogenic secondary metabolite produced by fungus, primarily Aspergillus flavus (Schmidt, 2013). Aflatoxin is often found in maize and peanuts (Leslie et al., 2008). A. flavus is likely to infect commercial maize in high heat and moist conditions due to poor crop management and inappropriate storage. Short-term exposure to high-dose aflatoxin can cause acute hepatitis, while long-term exposure can induce liver cancer (Wu and Khlangwiset, 2010). According to the United States Centers for Disease Control and Prevention (CDC), 4.5 billion people worldwide have been chronically exposed to aflatoxin. Moreover, 15,000 to 25,000 liver cancer cases annually are associated with chronic aflatoxin exposure in Asia and sub-Saharan Africa (Schmidt, 2013). Aflatoxin is a wellknown public health threat because it caused multiple toxic tragedies in Africa, notably the massive outbreak in Kenya in 2004. Therefore, strict regulation of crop management and preventative community education are essential to prevent future aflatoxin outbreaks.

Address correspondance to:

'Department of Environmental Toxicology, University of California-Davis. 1 Shields Avenue, Davis, CA 95616, United States of America

*ktan@ucdavis.edu

doi:10.22186/jyi.38.2.10-12

Accepted date: April 2020

Publication date: August 2020

\section{THE HISTORY AND BIOLOGICAL MECHANISM OF AFLATOXIN}

Aflatoxin was first discovered in the United Kingdom in 1960, when many turkeys suddenly died as a result of an unknown "Turkey X Disease", which was later attributed to the consumption of moldy peanuts. Investigation showed that the mold on the peanuts was the toxic $A$. flavus. There are three classes of aflatoxins. Aflatoxins $B$ and $G$ are produced by A. flavus, while aflatoxin $\mathrm{M}$ is a metabolite of aflatoxin $\mathrm{B}$. Aflatoxin $M$ is produced by the liver and stored in the milk of humans and animals. The most prominent type of aflatoxin is aflatoxin B1. After ingestion of aflatoxin-contaminated maize, aflatoxin B1 is converted by hepatic CYP450 enzymes into aflatoxin B1 exo-8,9-epoxide, a more toxic molecule (Schmidt, 2013). Even though CYP450 enzymes are often associated with detoxification, they activate aflatoxin to become an electrophile to generate DNA adducts. Aflatoxin forms DNA adducts by interacting its exo-epoxide with DNA and therefore induces genetic mutation of the tumor suppressor gene p53, thus increasing risk of cancer (Leslie et al., 2008). This is especially true in individuals who are infected by Hepatitis B Virus (HBV) and are chronically exposed to aflatoxin. These individuals are 30-times more likely to contract liver cancer when compared to individuals not infected with HBV (Magnussen and Parsi, 2013).

\section{TOXIC TRAGEDY: AFLATOXIN OUTBREAK IN KE- NYA (2004)}

The most severe aflatoxin outbreak documented to date occurred in Kenya in 2004. The outbreak occurred in the Makueni District, a district with a population of 771,545 which previously had another aflatoxin outbreak in 1981 . This outbreak resulted in 317 cases and 125 deaths (Nyikal et al., 2004). Patients presented with abdominal pain, pulmonary edema, and liver necrosis after having ingested $50 \mathrm{mg}$ of aflatoxin B1 per day. These patients were later diagnosed with aflatoxicosis. Aflatoxin caused suffering in patients ranging from 1 to 83 years of age, with an average age of 22 . The source of this aflatoxin outbreak was attributed primarily to local aflatoxin-contaminated maize. The aflatoxin level found 
in the maize frequently exceeded $5000 \mathrm{ng} / \mathrm{g}$, far higher than the limit of less than $20 \mathrm{ng} / \mathrm{g}$ established by European and North American health authorities (Leslie et al., 2008). Although Kenya previously underwent an aflatoxin outbreak in 1982, the 2004 outbreak was unparalleled in its magnitude of severe economic loss, food shortage, and gross medical cases. By its conclusion, Kenya had lost millions of dollars due to this outbreak, and this severe loss created barriers for future agricultural trade (Leslie et al., 2008). As a result of the massive human and financial cost of the outbreak, the World Health Organization (WHO) has since raised awareness to control aflatoxin and its associated diseases (Mutegi et al., 2018).

Post-outbreak investigations have identified several factors that caused the outbreak. First, the corn maize produced in and imported to Kenya was stored in a warm and humid environment, which facilitates fungal growth. A. flavus growth is heat-resistant, even at temperatures as high as $118^{\circ} \mathrm{F}\left(87^{\circ} \mathrm{C}\right)$, and high humidity levels favor fungal growth. The lack of mechanical drying of the crops to avoid humidity increased the risk of fungal infection (Schmidt, 2013). Other factors such as seasonal rain and flood can influence maize storage temperature and humidity levels. Further investigation found that the early arrival of rain in February 2004 caused an early maize harvest. Without proper storage sites, most farmers stored the maize in their homes, with leaking roofs. Both the 1981 and 2004 outbreaks were preceded by a period of drought and unexpected rain during the harvest season, which may have contributed to favorable fungal growth in the household maize (Lewis et al., 2005). The extreme weather conditions, in addition to a lack of artificial crop drying techniques, provided a moist medium that allowed $A$. flavus to colonize the maize and produce aflatoxin.

Furthermore, lack of diet variety contributed significantly to the breadth of Kenya's 2004 aflatoxin outbreak. Most victims of this tragedy were of lower socioeconomic status, their diets consisting primarily of maize (Leslie et al., 2008). The extended drought period in Kenya caused food resources to be scarce, forcing these disadvantaged citizens to consume aflatoxin-contaminated maize unknowingly. Moreover, the typical shortage of maize product drove farmers to sell the clean, uninfected maize and leave the contaminated maize for their own consumption (Leslie et al., 2008). This led to a widespread distribution of aflatoxin-contaminated maize in the local market. Unaware, market vendors sold the contaminated maize to wider areas, expanding the geographic range of aflatoxin exposure and ingestion. Additionally, the contaminated maize remained on the market even after the contaminated household maize was disposed of by local public health authorities (Lewis et al., 2005). Since consumers in Kenya have a diet largely dependent on maize, the immediate toxicological effects were amplified. This dependence on maize may likely have caused chronic exposure to aflatoxin, which increases liver cancer incidence (Schmidt, 2013).

Another crucial factor contributing to this aflatoxin tragedy was the lack of medical resources in Kenya. Many Kenyans do not have convenient access to healthcare, often needing to travel several hours to consult with a healthcare professional. Patients of low socioeconomic status also contended with decreased access to healthcare (Mutegi et al., 2018). Consequently, in 2004, many people did not seek medical assistance quickly enough in the outbreak and died of aflatoxicosis before they arrived at a hospital. Many of the documented statistics may even be underestimates due to the large number of unreported deaths of those who did not have access to healthcare (Leslie et al., 2008). The limited medical resources, as well as a lack of communication between medical, public health, and agricultural stakeholders prevented effective and timely control of the aflatoxin outbreak.

\section{LESSONS LEARNED AND POTENTIAL SOLU- TIONS}

Modern agricultural innovations and public health improvements can be helpful in preventing future outbreaks and their clearly devastating consequences. Proper drying and storage conditions can directly reduce the risk of aflatoxin outbreak by preventing $A$. flavus growth. Strict quality control of harvested maize cane, through identification and elimination of the moldy maize. This can involve measures to ensure that drying occurs in environments under $12 \%$ humidity before storage, to prevent $A$. flavus growth (Wagacha and Muthomi, 2008). Examples of large-scale agricultural implementation to reduce $A$. flavus colonization include bulk grain driers, massive storage sites, and aircraft pesticide application. Recent efforts included the AflaSTOP project initiated by local public-sector institutions. This aims to provide cost-effective storage technologies, such as the EasyDry M500 portable dryer, to reduce maize humidity (Mutegi et al., 2018). High crop quality not only maximizes resource efficiency, but also increases revenue to employ workers and fund technological innovations to screen for damaged kernels. Since crop trade is a major source of income in Kenya, investing in crop quality control can increase profits from crop harvest and export (Schmidt, 2013).

In addition to quality control at the agricultural scale, biocontrol can occur at a molecular level. Peter Cotty, a plant pathologist at the USDA, proposed to inoculate maize in the field with atoxigenic strains of $A$. flavus early in their life cycle. This protects them from fungal colonization and mycotoxin production by outcompeting the toxic strains of $A$. flavus (Schmidt, 2013). The analogous fungus, Aflasafe, is currently used as a biocontrol in Nigeria. Aflasafe has reduced aflatoxin contamination in nuts and corns by $80 \%$ to $90 \%$ (Schmidt, 2013). Additionally, Kenya has benefitted from the regional leadership of Partnership for Aflatoxin Control in Africa (PACA). They have helped develop infrastructure and 
technologies to mitigate aflatoxin from crops and pursued agricultural research to find a more effective and permanent solution to control aflatoxin presence in crop fields. Possible future research initiatives can regulate maize growth via plant immunity against $A$. flavus colonization. This strategy of $A$. flavus growth inhibition in plants is powerful because it minimizes aflatoxin production (Mutegi et al., 2018).

Governments have yet to establish strict regulations in detecting and removing aflatoxin-contaminated crops through the development of agricultural policies. These can help farmers communicate their concerns over crop quality and recover from the loss of aflatoxin-contaminated crops. In order to increase food security and control food quality, designated officials such as Kenya's National Cereals and Produce Board (NCPB) could potentially supervise the collection and distribution of corn maize to implement removal of damaged maize. Centralizing the rural maize market can increase surveillance and documentation of maize quality, thereby reducing the risk of aflatoxin contamination (Leslie et al., 2008). Seminars conducted by government agencies could report current harvest conditions and assess the possibility of an aflatoxin outbreak among local communities. Collaborations with researchers would also provide opportunities to battle against aflatoxin and fungal infection (Wagacha et al., 2008). When powerful authorities implement policy changes, administrative and legislative stakeholders could exert forces to minimize risk of aflatoxin outbreak by maintain a high crop quality standard.

Another effective disease management protocol is increased health education of local citizens. Healthcare officials can more heavily advocate for a diet that is composed of multiple grains instead of mostly maize. Financial incentives for consuming a diverse diet composed of multiple grains, such as rice discount coupons, could minimize the risk of aflatoxin-contaminated maize consumption. Government funding could sponsor these financial assistance programs to promote a healthier diet style. Long-term monitoring of aflatoxin levels on susceptible populations through blood tests can provide active surveillance to detect outbreaks early if local health authorities would provide funding (Nyikal et al., 2004). While screening for aflatoxin toxicity is financially costly, emphasis on routine healthcare checkups can detect signs of early aflatoxicosis, consequently improving population health while lowering the prevalence of aflatoxin outbreak. Moreover, ongoing educational campaigns by the local public health authorities on how to identify aflatoxin-contaminated crops can increase awareness among villagers and farmers to select and consume maize carefully. Distributing information about aflatoxicosis symptoms and risk factors to local citizens enables them to seek medical help immediately once these symptoms appear (Mutegi et al., 2018).

\section{CONCLUSION}

Long-term exposure and ingestion of aflatoxin are associated with the development of liver cancer. The aflatoxin outbreak that occurred in Kenya in 2004 revealed problems in public health management as well as agricultural procedures in the processing and storage of crops. Fortunately, modern agricultural technologies and advances in preventive medical care could further enhance both crop quality control and aflatoxin-associated disease management. The development of agriculture technology such as Aflasafe and EasyDry M500 has led to safer processing and storage conditions for crops. However, local health authorities could install periodic checks on aflatoxin levels in crops sold in market to monitor the potential for possible outbreaks in addition to government funding to educate patients on aflatoxin and safe consumption of crops. Increasing accessibility and quality of healthcare by regularly checking blood for aflatoxin levels can further surveillance of the population's health, if financial support is viable. With substantial efforts from various stakeholders such as government leaders and local public health officers, as well as progress in scientific research, authorities can enhance aflatoxin control policies and prevent additional outbreaks in the future.

\section{ACKNOWLEDGMENTS}

The author would like to thank Dr. Robert Rice (Professor and Chair of the Department of Environmental Toxicology at University of California-Davis) for supporting this research project and providing feedback on early versions of this manuscript.

\section{REFERENCES}

Leslie, J., Bandyopadhyay, R., and Visconti, A. (2008). Mycotoxins: detection methods, management, public health and agricultural trade. Wallingford: $C A B$ International.

Lewis, L., Onsongo, M., Njapau, H., Schurz-Rogers, H., Luber, G., Kieszak, S., Nyamongo, J., Backer, L., Dahiye, A. M., Misore, A., DeCock, K., Rubin, C., and Kenya Aflatoxicosis Investigation Group (2005). Aflatoxin contamination of commercial maize products during an outbreak of acute aflatoxicosis in eastern and central Kenya. Environmental health perspectives, 113(12), 1763-1767.

Magnussen, A. and Parsi, M. A. (2013). Aflatoxins, hepatocellular carcinoma and public health. World Journal of Gastroenterology, 19(10), 1508-12.

Mutegi, C., Cotty, P. and Bandyopadhyay, R. (2018). Prevalence and mitigation of aflatoxins in Kenya (1960 - to date). World Mycotoxin Journal, 11(3), 341-357. doi: 10.3920/wmj2018.2362.

Outbreak of Aflatoxin Poisoning - Eastern and Central Provinces, Kenya, January - July 2004. (2004). Morbidity and Mortality Weekly Report, 53(34), 790-793.

Schmidt, C. W. (2013). Breaking the mold: new strategies for fighting aflatoxins. Environmental Health Perspectives, 121(9), A270-5.

Wagacha, J. M. and Muthomi, J. W. (2008). Mycotoxin problem in Africa: Current status, implications to food safety and health and possible management strategies. International Journal of Food Microbiology, 124(1), 1-12.

Wu, F. and Khlangwiset, P. (2010). Health economic impacts and cost-effectiveness of aflatoxin-reduction strategies in Africa: case studies in biocontrol and post-harvest interventions. Food additives \& contaminants. Part A, Chemistry, analysis, control, exposure \& risk assessment, 27(4), 496-509. doi: 10.1080/19440040903437865 